\title{
Associação entre a abordagem médica centrada na pessoa e a satisfação com a consulta em atenção primária à saúde
}

\author{
Association between the person-centered medical approach and satisfaction with the \\ consultation in primary health care
}

\section{Asociación entre el enfoque médico centrado en la persona y la satisfacción con la consulta en atención primaria de salud}

Rodrigo Caprio Leite de Castro ${ }^{\circledR}$, Daniela Riva Knauth ${ }^{\circledR}$

Faculdade de Medicina, Universidade Federal do Rio Grande do Sul, Porto Alegre, RS, Brasil

\section{Resumo}

Introdução: A aplicação do método clínico centrado na pessoa (MCCP) busca alcançar a compreensão da pessoa e da sua doença por meio da abordagem de duas perspectivas: a do médico e a da pessoa. A satisfação dos usuários é considerada um importante indicador de avaliação dos serviços. Objetivo: O presente artigo tem como objetivo investigar se há associação entre o grau de orientação ao MCCP, segundo a percepção da pessoa, e a satisfação com a última consulta médica em um serviço de atenção primária à saúde (APS). Metodologia: Trata-se de estudo transversal, realizado com pessoas com hipertensão e/ou diabetes nas 12 unidades de um serviço de APS, em Porto Alegre, RS $(n=408)$. O grau de orientação ao MCCP foi medido pelo instrumento "Percepção do Paciente sobre o Centramento da Consulta" (PPCC). Resultados: O baixo escore geral do PPCC (o que corresponde à alta orientação ao MCCP) esteve associado significativamente com a maior satisfação das pessoas com aspectos relacionados ao serviço (forma de agendamento e cordialidade da recepção) e ao atendimento médico (atenção dada às queixas pelo médico, exame físico do médico, explicações sobre problema e explicações sobre prognóstico), com a consulta em geral e com o tratamento da hipertensão arterial sistêmica e/ou do diabetes mellitus na unidade de saúde. Discussão: $O$ médico de APS deve ser mais orientado à pessoa para que possa atingir, portanto, níveis mais altos de satisfação. Nessa perspectiva, a incorporação da satisfação dos usuários na avaliação dos serviços de saúde deve se constituir em indicador de qualidade, sobretudo, da abordagem praticada pelo médico.

Palavras-chave: Assistência Centrada no Paciente; Satisfação do Paciente; Indicadores de Qualidade em Assistência à Saúde; Atenção Primária à Saúde

Como citar: Castro RCL, Knauth DR. Associação entre a abordagem médica centrada na pessoa e a satisfação com a consulta em atenção primária à saúde. Rev Bras Med Fam Comunidade. 2021;16(43):2702. https://doi.org/10.5712/rbmfc16(43)2702

\author{
Autor correspondente: \\ Rodrigo Caprio Leite de Castro. \\ E-mail: rcastro@hcpa.edu.br \\ Fonte de financiamento: \\ parcial pelo PPG em Epidemiologia da \\ FAMED/UFRGS. \\ Parecer CEP: \\ GHC: 421.153 / UFRGS: 400.164 \\ Procedência: \\ não encomendado. \\ Avaliação por pares: \\ externa. \\ Recebido em: 06/09/2020. \\ Aprovado em: 07/01/2021.
}




\begin{abstract}
Introduction: The application of the patient-centered clinical method (PCCM) aims to achieve the understanding about the patient and his/her condition by means of the approach from two perspectives: the doctor's and the patient's. The satisfaction of the users is considered as a major indicator of service evaluation. Purpose: This paper aims to investigate whether there is an association between the degree of PCCM orientation, according to the patient's perception, and the satisfaction with the most recent medical appointment in a primary health care (PHC) service. Methods: This is a cross-sectional study, developed with hypertensive and/or diabetic patients in the 12 health care centers of a PHC service in Porto Alegre, RS $(n=408)$. The degree of PCCM orientation was measured by the use of the "Patient Perception of Patient-Centeredness" (PPPC) tool. Results: The low general score of the PPPC (corresponding to the high PCCM orientation) was significantly associated with the higher satisfaction of the patients with aspects related to the service (scheduling mode and cordiality at the reception) and the medical care (attention paid to the demands by the doctor, physical exam by the doctor, explanations about the problem, and explanations on the prognosis), the appointment in general, and to the systemic hypertension and/or diabetes mellitus treatment in the health care center. Discussion: The PHC doctor must be more person-oriented to achieve, therefore, higher levels of satisfaction. In this perspective, the incorporation of the users' satisfaction in the evaluation of health services must be a quality indicator, especially of the approach practiced by the doctor.
\end{abstract}

Keywords: Patient-Centered Care; Patient Satisfaction; Quality Indicators, Health Care; Primary Health Care

\title{
Resumen
}

Introducción: La aplicación del método clínico centrado en la persona (MCCP) busca lograr la comprensión del paciente y su enfermedad a través del abordaje desde dos perspectivas: la del médico y la del paciente. La satisfacción del usuario se considera un indicador importante de la evaluación del servicio. Objetivo: Este artículo tiene como objetivo investigar si existe una asociación entre el grado de orientación al MCCP, según la percepción del paciente, y la satisfacción con la última consulta médica en un servicio de atención primaria de salud (APS). Métodos: Se trata de un estudio transversal, realizado con pacientes hipertensos y/o diabéticos en las 12 unidades de un servicio de APS, en Porto Alegre, RS ( $n=408)$. El grado de orientación al MCCP se midió mediante el instrumento "Percepción del Paciente en el Centro de Consulta" (PPCC). Resultados: La baja puntuación general del PPCC (que corresponde a la alta orientación al MCCP) se asoció significativamente con la mayor satisfacción de los pacientes con aspectos relacionados con el servicio (forma de programación y cordialidad de recepción) y la atención médica (atención de las quejas por parte del médico), exploración física por parte del médico, explicaciones del problema y explicaciones de pronóstico), con consulta general y tratamiento de hipertensión arterial sistémica y/o diabetes mellitus en la unidad de salud. Discusión: El médico de la APS debe estar más orientado a las personas para que así pueda alcanzar mayores niveles de satisfacción. En esta perspectiva, la incorporación de la satisfacción del usuario en la valoración de los servicios de salud debe ser un indicador de calidad, sobre todo, del abordaje practicado por el médico.

Palabras clave: Atención Dirigida al Paciente; Satisfacción del Paciente; Indicadores de Calidad de la Atención de Salud; Atención Primaria de Salud

Na década de 1980, pesquisadores do “Grupo de Comunicação Médico-Paciente”, da Universidade de Western Ontario, Canadá, com base em um entendimento multidimensional da abordagem médica, sistematizaram o método clínico centrado na pessoa (MCCP) $)^{1,2}$.

O MCCP busca alcançar a compreensão da pessoa e da sua doença por meio da abordagem de duas perspectivas, a da pessoa e a do médico. O médico acessa a perspectiva da pessoa tanto pela recepção dos seus sinais, suas dicas e sugestões, como também pelo encorajamento à expressão dos seus sentimentos, ideias e expectativas, oferecendo, por sua vez, a sua própria visão ou a sua explicação para a doença à pessoa. Ambas as perspectivas devem, então, ser abordadas e os potenciais conflitos tratados por negociação. Nesse sentido, o MCCP contrasta-se com o método clínico centrado na doença, em que, segundo os autores, apenas a perspectiva do médico é abordada ${ }^{1,2}$.

De acordo com Stewart et al. ${ }^{3}$, o MCCP compreende quatro componentes interativos, a saber: "explorando a saúde, a doença e a experiência da doença", "entendendo a pessoa como um todo", "elaborando um plano conjunto de manejo dos problemas" e "intensificando a relação entre pessoa e médico". O objetivo último do MCCP é a elaboração do plano conjunto de manejo dos problemas que deve ser satisfatório tanto para a pessoa que busca cuidado em saúde quanto para o médico. 
A satisfação pode ser definida em termos das expectativas e da percepção que os usuários têm dos serviços recebidos ${ }^{4}$. A satisfação dos usuários vem sendo considerada um importante indicador de avaliação dos serviços ${ }^{5,6}$, podendo, primeiro, ser utilizada na orientação de medidas corretivas, por meio da identificação de pontos críticos a serem melhorados, e, segundo, estar potencialmente relacionada à melhora na adesão ao tratamento, à relação médico-pessoa e à maior adequação no uso do serviço ${ }^{7,8}$.

Existem, na literatura, estudos bem delineados que exploram a associação entre a abordagem médica centrada na pessoa e a satisfação da pessoa com a consulta. Os estudos de Kinnersley et al. ${ }^{10}$, Little et al. ${ }^{11}$, Flocke, Miller e Crabtree ${ }^{12}$ e Rohrer et al. ${ }^{13}$ encontram associação entre a maior orientação à pessoa e a maior satisfação com a consulta. Contudo, a associação entre ambas não foi confirmada nos estudos de Stewart ${ }^{14}$, Mead, Bower e Hann ${ }^{15}$ e Bertakis e Azari ${ }^{16}$, revelando, assim, resultados contraditórios.

Frente a este contexto, o presente estudo tem como objetivo investigar se o grau de orientação ao MCCP, segundo a percepção da pessoa, encontra-se associado à satisfação do usuário com a última consulta médica.

\section{Metodologia}

Este estudo foi realizado nas 12 unidades de um serviço de APS de Porto Alegre, RS, no período compreendido entre novembro de 2013 e novembro de 2014.

O delineamento é transversal e a população de pesquisa é constituída pelas pessoas maiores de 18 anos inscritos no Programa de Hipertensos e Diabéticos (Hiperdia) do referido serviço. A amostra foi calculada considerando os dados de Mead, Bower e Hann ${ }^{15}$, poder de $80 \%$ e alfa de $5 \%$, chegando-se a um número amostral de 126 pacientes. No entanto, como esse estudo faz parte de um projeto maior em que outros desfechos foram também pesquisados, optamos por manter o maior número amostral necessário pelos desfechos em questão, de maneira que, o presente estudo, é realizado com 408 entrevistas. Os cálculos foram realizados com auxílio do software WinPepi.

Foram incluídos as pessoas maiores de 18 anos, inscritos no Hiperdia, com consulta médica marcada para a semana seguinte e duas ou mais consultas realizadas nos últimos dezoito meses com o mesmo médico da consulta agendada. Esse último critério teve como finalidade assegurar um vínculo mínimo entre o médico e a pessoa atendida. Foram excluídos as pessoas sem capacidade física e/ou mental para responderem o questionário e aqueles portadores de diabetes mellitus tipo I.

Foram entrevistados 34 pessoas/unidade (408 pessoas/12 unidades). Os critérios de elegibilidade foram verificados por meio da revisão dos prontuários das pessoas, cadastradas no Hiperdia, com consulta médica marcada para a semana seguinte. As pessoas foram entrevistadas após a consulta com o médico, na unidade de saúde. Incluiram-se, no estudo, as 34 consultas consecutivas de cada unidade que preencheram os critérios de inclusão - independentemente do médico, do dia da semana ou do turno de marcação (manhã ou tarde). Ao longo das 408 entrevistas consecutivas realizadas, houve 99 faltas às consultas e 32 recusas em participar da pesquisa. As faltas das pessoas às consultas e as recusas foram repostas em consultas consecutivas, seguindo-se o agendamento. $\mathrm{Na}$ análise do perfil das pessoas que faltaram ou recusaram participar da pesquisa, conforme as informações coletadas nos prontuários, observou-se semelhança entre as duas populações: a dos que faltaram ou recusaram e a dos respondentes. 
O instrumento foi dividido em três partes. Na primeira parte, para estudo do perfil dos sujeitos, aplicouse um questionário estruturado com questões sociodemográficas, econômicas e referentes à situação de saúde e utilização dos serviços, incluindo data de nascimento, sexo, cor da pele, situação conjugal, renda individual e familiar, escolaridade, situação ocupacional, presença de doenças crônicas, tempo de vínculo com o médico e com a unidade de saúde.

Na segunda parte, foi aplicado o instrumento "Percepção do Paciente sobre o Centramento da Consulta" (PPCC), que mede a orientação ao MCCP atribuída, pelo paciente, à última consulta realizada com o médico. O instrumento PPCC, validado por Stewart et al. ${ }^{17}$, é constituído de 14 questões. As quatro primeiras são referentes à medida do primeiro componente do MCCP, "explorando a doença e a experiência da doença"; as nove seguintes (da quinta até a décima terceira questão do instrumento), ao terceiro, "elaborando um plano conjunto de manejo dos problemas", e a última questão, ao segundo componente, "entendendo a pessoa como um todo". Para as respostas, utiliza-se uma escala Likert, graduada em quatro itens. A média aritmética das respostas atribuídas às 14 questões é igual ao escore geral do PPCC. Da mesma forma, a média aritmética das respostas atribuídas às quatro primeiras questões é igual ao escore do primeiro componente; a média aritmética das respostas conferidas às nove perguntas seguintes (da quinta até a décima terceira) é igual ao escore do terceiro componente e a resposta indicada ao décimo quarto item é igual ao escore do segundo componente. O PPCC foi validado, no Brasil, por Kolling e Harzheim ${ }^{18}$.

Na terceira e última parte, a satisfação dos usuários com relação à última consulta foi medida pelo instrumento validado por Kloetzel et al. ${ }^{7}$, que mede a satisfação com relação à facilidade de acesso a consulta, ao tempo despendido na sala de espera, à cordialidade por parte da recepção, à cordialidade do profissional, à atenção dada as queixas, à impressão do exame clinico, à confiança despertada pelo profissional, à confiança na receita, às explicações do médico com respeito à doença e quanto ao prognóstico, à satisfação com o agendamento e geral da consulta. O instrumento é constituído por escala Likert, graduada em cinco itens, do maior (letra A) ao menor (letra E) grau de satisfação, representados por figuras de faces com cinco expressões distintas de satisfação. Os entrevistados foram convidados a responder as perguntas baseando-se na experiência da última consulta. Para a análise, os resultados foram agrupados em duas classificações, sendo "satisfeito" quando referido "muito bom" ou "bom" (carinhas A ou B) e "insatisfeito" quando referido algum dos demais itens. Perguntou-se também acerca da satisfação com relação ao manejo da hipertensão arterial sistêmica (HAS) e do diabetes mellitus (DM) na unidade de saúde.

O coordenador de campo revisou os prontuários (na época do estudo, em papel), sendo o responsável pela inclusão das pessoas no estudo. As entrevistas foram realizadas por 9 entrevistadores, estudantes de graduação da área da saúde, treinados. Para a coleta de dados, utilizaram-se netbooks, tendo sido usado o software Le Sphinx Plus. O treinamento dos entrevistadores se deu por meio de estudo piloto e o seu acompanhamento foi realizado pelo coordenador de campo com observação presencial de entrevistas ao longo da coleta de dados.

As análises estatísticas foram realizadas com nível de significância de $5 \%$, bicaudal. Os dados são apresentados por meio de médias e desvios-padrão, de medianas e intervalos interquartílicos, de proporções e de respectivos intervalos de confiança. A medida da percepção do grau de orientação ao MCCP foi calculado por meio de escores em uma escala de 1 (que equivale a mais alta orientação ao 
MCCP) a 4 (que equivale a mais baixa orientação ao MCCP). Utilizou-se a mediana como ponto de corte entre a mais alta e a mais baixa orientação ao MCCP. Dessa forma, o baixo escore geral do PPCC (para valores pertencentes ao intervalo entre 1 e a respectiva mediana) corresponde à alta orientação ao MCCP e o alto escore geral do PPCC (para valores pertencentes ao intervalo entre a respectiva mediana e 4), à baixa orientação ao MCCP.

Para comparar a proporção de pessoas satisfeitas que atribuíram baixo e alto escore aos três componentes e ao escore geral do PPCC (o que corresponde à alta e à baixa orientação ao MCCP respectivamente), utilizou-se o teste qui-quadrado de Pearson com valor-p calculado por correção de continuidade de Yates ou por exato de Fisher. Essas análises foram realizadas no IBM SPSS Statistics 18.

Este projeto foi aprovado pelos Comitês de Ética das instituições envolvidas no estudo (número de protocolo 08147413.1.0000.5347). Somente foram realizadas entrevistas com as pessoas e médicos que aceitaram participar e assinaram o Termo de Consentimento Livre e Esclarecido.

\section{Resultados}

Dentre os 408 entrevistados das unidades de saúde investigadas, observa-se que a maioria deles tem mais de 60 anos (61\%), é do sexo feminino (72,5\%) e é de cor branca (73,3\%). Com relação à escolaridade, $25,7 \%$ afirmaram ter de 1 a 4 anos de estudo e $38,7 \%$, de 5 a 8 , sendo que somente 2,2\% disseram ter mais de 11 anos de estudo (ensino superior), e à renda mensal, 92,7\% dos entrevistados recebiam até 3 salários mínimos (SM) e 92,8\% das famílias somavam até 5 SM por mês, caracterizando, assim, uma população de baixa escolaridade e de baixa renda. A Tabela 1 apresenta o perfil dos entrevistados.

Dentre os participantes do estudo, 382 eram hipertensos, sendo que destes 115 também diabéticos, e 26 eram somente diabéticos. A maioria $(57,1 \%)$ não relatou diagnóstico de lesão em órgão alvo e $27,9 \%$ relatou apenas um desses diagnósticos. O tempo de acompanhamento médio com o médico com quem a pessoa realizou a consulta foi menor em comparação com o tempo médio de acompanhamento na unidade de saúde (6,0 e 13,7 anos respectivamente).

$\mathrm{Na}$ Tabela 2, são apresentadas as medianas, com os respectivos intervalos interquartílicos, dos escores dos três primeiros componentes do PPCC e do escore geral. Na Tabela 3, são descritas as proporções de respostas em cada uma das alternativas da escala de medida da satisfação por item perguntado. Chama atenção o fato de que os aspectos relacionados com o serviço de APS, como a facilidade de acesso, a forma de agendamento, o tempo despendido na sala de espera e a cordialidade da recepção, apresentaram os percentuais mais baixos de grau de satisfação (em comparação com os percentuais dos aspectos relacionados com o atendimento médico).

As Tabelas 4, 5, 6 e 7 mostram as proporções de pessoas satisfeitas com aspectos da última consulta e com o tratamento de HAS e/ou DM na unidade de saúde que atribuíram baixo e alto escore dos componentes 1 ("explorando a saúde, a doença e a experiência da doença"), 2 ("entendendo a pessoa como um todo") , 3 ("elaborando um plano conjunto de manejo dos problemas") e do escore geral do PPCC respectivamente. Destaca-se que as proporções de satisfeitos com a consulta (satisfação geral) e com o tratamento da HAS e/ou DM recebido na unidade de saúde que atribuíram baixo escore geral do PPCC ao médico (o que corresponde à alta orientação ao MCCP praticada por esse) foram significativamente maiores 
Tabela 1. Características da amostra segundo variáveis sócio-demográficas, econômicas, relativas à situação de saúde e à utilização de um serviço de APS de Porto Alegre, RS, 2015.

\begin{tabular}{|c|c|c|}
\hline Variáveis & n (\%) & média (DP) \\
\hline Idade & & $63,8(12,1)$ \\
\hline $18-40$ anos & $14(3,4)$ & \\
\hline $41-60$ anos & $145(35,6)$ & \\
\hline$>60$ anos & $249(61,0)$ & \\
\hline Sexo feminino & $296(72,5)$ & \\
\hline Cor branca & $299(73,3)$ & \\
\hline \multicolumn{3}{|l|}{ Situação conjugal } \\
\hline Casado & $201(49,3)$ & \\
\hline Viúvo & $100(24,5)$ & \\
\hline Separado & $59(14,5)$ & \\
\hline Solteiro & $48(11,8)$ & \\
\hline \multicolumn{3}{|l|}{ Sabe ler e escrever } \\
\hline Sim & $375(91,9)$ & \\
\hline Não & $33(8,1)$ & \\
\hline \multicolumn{3}{|l|}{ Escolaridade (anos de estudo) } \\
\hline \multicolumn{3}{|c|}{ (dos que referiram saber ler e escrever) } \\
\hline 0 & $14(3,4)$ & \\
\hline $1-4$ & $105(25,7)$ & \\
\hline $5-8$ & $158(38,7)$ & \\
\hline $9-11$ & $89(21,8)$ & \\
\hline$>11$ & $9(2,2)$ & \\
\hline \multicolumn{3}{|l|}{ Situação ocupacional } \\
\hline Desempregado & $17(4,2)$ & \\
\hline Empregado com carteira & $38(9,3)$ & \\
\hline Empregado sem carteira & $8(2,0)$ & \\
\hline Autônomo & $44(10,8)$ & \\
\hline Dona de casa & $44(10,8)$ & \\
\hline Aposentado & $207(50,7)$ & \\
\hline Pensionista & $27(6,6)$ & \\
\hline Auxílio doença & $22(5,4)$ & \\
\hline Seguro desemprego & $1(0,2)$ & \\
\hline \multicolumn{3}{|l|}{ Renda individual no último mês } \\
\hline \multicolumn{3}{|l|}{ (bruta) em números de SM } \\
\hline até $1 \mathrm{SM}$ & $186(48,6)$ & \\
\hline $1<\mathrm{SM} \leq 3$ & $169(44,1)$ & \\
\hline $3<\mathrm{SM} \leq 5$ & $26(6,8)$ & \\
\hline $5<\mathrm{SM} \leq 7$ & $2(0,5)$ & \\
\hline \multicolumn{3}{|l|}{ Renda familiar no último mês } \\
\hline \multicolumn{3}{|l|}{ (bruta) em números de SM } \\
\hline até $1 \mathrm{SM}$ & $43(11,8)$ & \\
\hline $1<\mathrm{SM} \leq 3$ & $205(56,5)$ & \\
\hline $3<\mathrm{SM} \leq 5$ & $89(24,5)$ & \\
\hline $5<\mathrm{SM} \leq 7$ & $16(4,4)$ & \\
\hline $7<\mathrm{SM} \leq 9$ & $7(1,9)$ & \\
\hline $\mathrm{SM}>9$ & $3(0,8)$ & \\
\hline
\end{tabular}




\begin{tabular}{lc}
...Continuação & \\
\hline Presença de hipertensão e/ou diabetes & $267(65,4)$ \\
Hipertensos somente & $26(6,4)$ \\
Diabéticos somente & \\
Hipetensos e diabéticos & \\
Presença de LOA & $60(14,7)$ \\
Insuficiência cardíaca & $9(2,2)$ \\
Cardiopatia isquêmica (sem IAM) & $22(5,4)$ \\
Cardiopatia isquêmica (com IAM) & $29(7,1)$ \\
Acidente vascular cerebral & $77(18,9)$ \\
Diminuição ou perda de visão & $51(12,5)$ \\
Perda de função renal & $1(0,2)$ \\
Amputação & \\
Número de LOA por pessoa & $233(57,1)$ \\
Nenhum & $114(27,9)$ \\
Um & $51(12,5)$ \\
Dois & $10(2,5)$ \\
Três ou mais
\end{tabular}

Tempo de acompanhamento na unidade de saúde (em anos)

$13,7(9,9)$

$6,0(6,9)$ (em anos)

$\overline{\mathrm{DP}}=$ desvio padrão. $\mathrm{SM}$ = salário mínimo. $\mathrm{LOA}$ = lesão em órgão alvo. IAM = infarto agudo do miocárdio.

Tabela 2. Escores medidos pelo instrumento "Percepção do Paciente sobre o Centramento da Consulta" (PPCC). Estudo em um serviço de APS de Porto Alegre, RS, 2015.

\begin{tabular}{lcc}
\hline Escore (1 - 4) & mediana & IIQ \\
\hline Escore do primeiro componente do MCCP & 1,00 & $1,00-1,50$ \\
Escore do segundo componente do MCCP & 1,00 & $1,00-1,00$ \\
Escore do terceiro componente do MCCP & 1,33 & $1,11-1,67$ \\
Escore total & 1,29 & $1,07-1,50$ \\
\hline
\end{tabular}

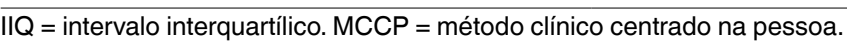

do que as dos que referiram alto escore (o que corresponde à baixa orientação ao MCCP praticada pelo médico). Essas diferenças se estenderam para as análises envolvendo os três componentes do MCCP, com exceção da que comparou a proporção de satisfeitos com a consulta (satisfação geral) com as dos que indicaram baixo e alto escore do componente 3, pois não mostrou significância estatística.

Com relação à satisfação com a atenção dada às queixas pelo médico, o exame físico do médico, as explicações sobre o problema e o prognóstico, demonstrou-se ser maior a satisfação nas pessoas que classificaram a abordagem do médico como sendo de baixo escore para os três compontentes e geral (o que significa maior orientação ao MCCP), sendo, portanto, as variáveis mais consistentemente envolvidas na relação positiva entre orientação à pessoa e satisfação com a consulta.

As outras variáveis acerca de aspectos relacionados com o médico não mostraram igual consistência. Com relação à cordialidade do médico e à confiança no médico, as proporções de satisfeitos que atribuíram 
Tabela 3. Satisfação com os serviços da unidade de saúde e com o médico. Estudo em um serviço de APS de Porto Alegre, RS, 2015.

\begin{tabular}{|c|c|c|c|c|c|}
\hline Variáveis (satisfação com) & $A(n \%)^{\star}$ & $B(n \%)^{*}$ & $C(n \%)^{*}$ & $D(n \%)^{*}$ & $E(n \%)^{*}$ \\
\hline \multicolumn{6}{|l|}{ Aspectos relacionados com o serviço } \\
\hline Facilidade de acesso & $229(56,1)$ & $99(24,3)$ & $49(12,0)$ & $22(5,4)$ & $9(2,2)$ \\
\hline Tempo despendido na sala de espera & $186(45,6)$ & $138(33,8)$ & $62(15,2)$ & $13(3,2)$ & $9(2,2)$ \\
\hline Cordialidade da recepção & $261(64,0)$ & $96(23,5)$ & $32(7,8)$ & $13(3,2)$ & $6(1,5)$ \\
\hline Atenção dada às queixas pelo médico & $346(84,8)$ & $57(14,0)$ & $2(0,5)$ & $1(0,2)$ & $2(0,5)$ \\
\hline Exame físico do médico & $304(74,5)$ & $93(22,8)$ & $6(1,5)$ & $2(0,5)$ & $3(0,7)$ \\
\hline Confiança no médico & $358(87,7)$ & $48(11,8)$ & $1(0,2)$ & - & $1(0,2)$ \\
\hline Confiança na receita & $363(89,0)$ & $44(10,8)$ & $1(0,2)$ & - & - \\
\hline Tratamento da HAS/DM na US & $266(65,2)$ & $125(30,6)$ & $12(2,9)$ & $3(0,7)$ & $2(0,5)$ \\
\hline
\end{tabular}

Tabela 4. Proporção de pessoas satisfeitas que atribuíram baixo escore ao componente 1, "explorando a doença e a experiência da doença" (o que significa maior orientação a esse componente). Estudo em um serviço de APS de Porto Alegre, RS, 2015.

\begin{tabular}{|c|c|c|c|}
\hline \multirow[b]{2}{*}{ Satisfeitos $(A+B)$ com } & \multicolumn{2}{|c|}{ Escore componente 1} & \multirow[b]{2}{*}{ Valor-p* } \\
\hline & $\begin{array}{c}\text { Menor orientação ao } \\
\text { componente } 1 \\
(>1,01) \\
n=202\end{array}$ & $\begin{array}{c}\text { Maior orientação ao } \\
\text { componente } 1 \\
(<=1,0) \\
n=206\end{array}$ & \\
\hline \multicolumn{4}{|l|}{ Aspectos relacionados com o serviço } \\
\hline Facilidade de acesso & 161 & 167 & 0,824 \\
\hline Tempo despendido na sala de espera & 155 & 169 & 0,229 \\
\hline Cordialidade da recepção & 172 & 185 & 0,203 \\
\hline \multicolumn{4}{|c|}{ Aspectos relacionados com o atendimento médico } \\
\hline Cordialidade do médico & 199 & 206 & 0,120 \\
\hline Atenção dada às queixas pelo médico & 197 & 206 & 0,029 \\
\hline Explicações sobre problema & 194 & 206 & 0,003 \\
\hline Explicações sobre prognóstico & 187 & 202 & 0,017 \\
\hline Geral com consulta & 190 & 203 & 0,032 \\
\hline Tratamento da HAS/DM na US & 188 & 203 & 0,012 \\
\hline
\end{tabular}

HAS = hipertensão arterial sistêmica. DM = diabetes mellitus tipo 2. US = unidade de saúde.

* Teste $\chi^{2}$ de Pearson, valor-p calculado por correção de continuidade de Yates ou por exato de Fisher. 
Tabela 5. Proporção de pessoas satisfeitas que atribuíram baixo escore ao componente 2, "entendendo a pessoa como um todo" (o que significa maior orientação a esse componente). Estudo em um serviço de APS de Porto Alegre, RS, 2015.

\begin{tabular}{|c|c|c|c|}
\hline \multirow[b]{2}{*}{ Satisfeitos $(A+B)$ com } & \multicolumn{2}{|c|}{ Escore componente 2} & \multirow[b]{2}{*}{ Valor-p* } \\
\hline & $\begin{array}{c}\text { Menor orientação ao } \\
\text { componente } 2 \\
(>2,0) \\
n=84\end{array}$ & $\begin{array}{c}\text { Maior orientação ao } \\
\text { componente } 2 \\
(<=1,0) \\
n=324\end{array}$ & \\
\hline \multicolumn{4}{|l|}{ Aspectos relacionados com o serviço } \\
\hline Facilidade de acesso & 58 & 270 & 0,005 \\
\hline Tempo despendido na sala de espera & 59 & 265 & 0,029 \\
\hline Cordialidade da recepção & 65 & 292 & 0,003 \\
\hline \multicolumn{4}{|c|}{ Aspectos relacionados com o atendimento médico } \\
\hline Cordialidade do médico & 81 & 324 & 0,008 \\
\hline Atenção dada às queixas pelo médico & 79 & 324 & $<0,001$ \\
\hline Explicações sobre problema & 77 & 323 & $<0,001$ \\
\hline Explicações sobre prognóstico & 71 & 318 & $<0,001$ \\
\hline Geral com consulta & 75 & 318 & 0,001 \\
\hline Tratamento da HAS/DM na US & 74 & 317 & $<0,001$ \\
\hline
\end{tabular}

HAS = hipertensão arterial sistêmica. DM = diabetes mellitus tipo 2. US = unidade de saúde.

* Teste $\chi^{2}$ de Pearson, valor-p calculado por correção de continuidade de Yates ou por exato de Fisher.

Tabela 6. Proporção de pessoas satisfeitas que atribuíram baixo escore ao componente 3, "elaborando um plano conjunto de manejo dos problemas" (o que significa maior orientação a esse componente). Estudo em um serviço de APS de Porto Alegre, RS, 2015.

\begin{tabular}{|c|c|c|c|}
\hline \multirow[b]{2}{*}{ Satisfeitos $(A+B)$ com } & \multicolumn{2}{|c|}{ Escore componente 3} & \multirow[b]{2}{*}{ Valor-p* } \\
\hline & $\begin{array}{c}\text { Menor orientação ao } \\
\text { componente } 3 \\
(>1,34) \\
n=169\end{array}$ & $\begin{array}{c}\text { Maior orientação ao } \\
\text { componente } 3 \\
(<=1,33) \\
n=239\end{array}$ & \\
\hline \multicolumn{4}{|l|}{ Aspectos relacionados com o serviço } \\
\hline Facilidade de acesso & 131 & 197 & 0,269 \\
\hline Tempo despendido na sala de espera & 133 & 191 & 0,861 \\
\hline Cordialidade da recepção & 139 & 218 & 0,011 \\
\hline \multicolumn{4}{|c|}{ Aspectos relacionados com o atendimento médico } \\
\hline Cordialidade do médico & 166 & 239 & 0,070 \\
\hline Atenção dada às queixas pelo médico & 164 & 239 & 0,012 \\
\hline Confiança na receita & 168 & 239 & 0,414 \\
\hline Explicações sobre problema & 161 & 239 & 0,001 \\
\hline Explicações sobre prognóstico & 154 & 235 & 0,002 \\
\hline Geral com consulta & 159 & 234 & 0,079 \\
\hline Tratamento da HAS/DM na US & 156 & 235 & 0,006 \\
\hline
\end{tabular}

HAS = hipertensão arterial sistêmica. $\mathrm{DM}=$ diabetes mellitus tipo 2. US = unidade de saúde.

* Teste $\chi^{2}$ de Pearson, valor-p calculado por correção de continuidade de Yates ou por exato de Fisher. 
Tabela 7. Proporção de pessoas satisfeitas que atribuíram baixo escore geral do PPCC (o que significa maior orientação à pessoa). Estudo em um serviço de APS de Porto Alegre, RS, 2015.

\begin{tabular}{|c|c|c|c|}
\hline \multirow[b]{2}{*}{ Satisfeitos $(A+B)$ com } & \multicolumn{2}{|c|}{ Escore geral } & \multirow[b]{2}{*}{ Valor-p* } \\
\hline & $\begin{array}{c}\text { Menor orientação à } \\
\text { pessoa } \\
(>1,30) \\
n=170\end{array}$ & $\begin{array}{c}\text { Maior orientação à } \\
\text { pessoa } \\
(<=1,29) \\
n=238\end{array}$ & \\
\hline \multicolumn{4}{|l|}{ Aspectos relacionados com o serviço } \\
\hline Facilidade de acesso & 130 & 198 & 0,119 \\
\hline Forma de agendamento & 141 & 221 & 0,003 \\
\hline Tempo despendido na sala de espera & 134 & 190 & 0,901 \\
\hline Cordialidade da recepção & 141 & 216 & 0,028 \\
\hline \multicolumn{4}{|c|}{ Aspectos relacionados com o atendimento médico } \\
\hline Cordialidade do médico & 167 & 238 & 0,072 \\
\hline Atenção dada às queixas pelo médico & 165 & 238 & 0,012 \\
\hline Exame físico do médico & 159 & 238 & $<0,001$ \\
\hline Confiança no médico & 168 & 238 & 0,173 \\
\hline Confiança na receita & 169 & 238 & 0,417 \\
\hline Explicações sobre problema & 162 & 238 & 0,001 \\
\hline Explicações sobre prognóstico & 154 & 235 & $<0,001$ \\
\hline Geral com consulta & 159 & 234 & 0,023 \\
\hline Tratamento da HAS/DM na US & 157 & 234 & 0,006 \\
\hline
\end{tabular}

HAS = hipertensão arterial sistêmica. $\mathrm{DM}=$ diabetes mellitus tipo 2. US = unidade de saúde.

* Teste $\chi^{2}$ de Pearson, valor-p calculado por correção de continuidade de Yates ou por exato de Fisher.

baixo e alto escore do PPCC somente foram diferentes significativamente nas análises do componente 2. Acerca da confiança na receita, nenhuma diferença entre proporções de satisfeitos foi verificada.

A respeito dos aspectos relacionados com o serviço de APS, as variáveis cordialidade da recepção e forma de agendamento foram as únicas que mostraram diferenças significativas entre as proporções de satisfeitos dos que atribuíram baixo e alto escore geral do PPCC. Todas as análises que envolveram esses aspectos da satisfação e o escore do componente 2 foram significativas, ao contrário do componente 1 , que não mostrou diferença entre os percentuais de satisfeitos em qualquer desses aspectos que indicaram baixo e alto escore. As proporções de satisfeitos com a cordialidade da recepção foram as únicas que demonstraram diferenças significativas entre os que atribuíram menor e maior escore do componente 3.

\section{Discussão}

Os resultados deste estudo evidenciam que as pessoas que atribuíram baixo escore geral do PPCC (o que significa alta orientação ao MCCP) à abordagem do médico apresentaram também maior satisfação geral com a última consulta médica, podendo-se afirmar assim que existe associação positiva entre o grau de orientação ao MCCP, vivenciado pela pessoa na consulta, e a sua satisfação com a mesma. Da mesma forma, os resultados apresentados sustentam que, nas pessoas com hipertensão e/ou diabetes, a maior orientação ao MCCP conferida por eles aos seus médicos associa-se com a maior satisfação a respeito do tratamento da HAS e/ou DM recebido na unidade de saúde. Essa associação acontece, em sua maior parte, em razão do MCCP favorecer, sobretudo, a prática de uma clínica culturalmente adequada. A prática 
dessa clínica pode ser compreendida mediante o conceito de competência cultural, que é, segundo Beach et al. ${ }^{19}$, a habilidade em se estabelecer uma comunicação interpessoal efetiva e uma relação capaz de superar as diferenças culturais existentes.

As variáveis atenção dada às queixas pelo médico, exame físico do médico, explicações sobre o problema e prognóstico mantiveram a associação entre a maior orientação ao MCCP e a maior satisfação com cada uma delas ao longo das análises realizadas com os escores dos três componentes do PPCC e do escore geral, permitindo-se, com isso, afirmar que este aspectos são os mais importantes implicados na relação entre o grau de orientação ao MCCP, segundo a percepção da pessoa, e a satisfação com a consulta.

Kloetzel et al. ${ }^{7}$, em pesquisa conduzida em uma unidade docente-assistencial de APS, em Pelotas, RS ( $n=135)$, que validou o instrumento de medida de satisfação utilizado, encontrou, de maneira geral, proporções semelhantes de pessoas satisfeitas $(A+B)$ com os aspectos do serviço e dos médicos que foram avaliados. As maiores diferenças com relação aos dados deste estudo mostram-se em relação aos itens facilidade de acesso (em Kloetzel et al., 65\% foram satisfeitos enquanto, neste, 80,4\%) e tempo despendido na sala de espera ( $43 \%$ versus $79,4 \%$ ), ambos aspectos relacionados com os serviços.

Os achados do presente estudo somam-se também àqueles evidenciados pelas pesquisas de Kinnersley et al. ${ }^{10}$, Little et al. ${ }^{11}$, Flocke, Miller e Crabtree ${ }^{12}$ e de Rohrer et al. ${ }^{13}$, que mostraram associação entre a maior orientação à pessoa e a satisfação com a consulta. Kinnersley et al. ${ }^{10}$, em estudo que teve como objetivo avaliar se a abordagem centrada na pessoa, praticada por médicos generalistas em unidades de APS do País de Gales, Reino Unido, se associava com melhores resultados das consultas ( $n=143$ ), demonstraram que a pontuação centrada na pessoa foi positiva e significativamente associada com a satisfação com a consulta. Little et al. ${ }^{11}$, em estudo observacional, realizado em três clínicas de APS inglesas, mostraram associação entre as características da MCP, estimada pelo instrumento "Medida de Cuidado Ambulatorial", em inglês Consultation Care Measure, "comunicação e parceria" (um médico simpático interessado em preocupações e expectativas dos pacientes e que discute e concorda sobre o problema e o tratamento) e "abordagem positiva" (ser positivo sobre o diagnóstico e o prognóstico) e a maior satisfação com a consulta.

Na pesquisa de Flocke, Miller e Crabtree ${ }^{12}$, realizada com 2881 pacientes de 138 médicos de família e comunidade de 84 unidades de APS de Ohio, Estados Unidos da América (EUA), mostrou-se que a satisfação das pessoas foi significativamente maior com a abordagem classificada como "focada na pessoa", em comparação com a "biomédica", "biopsicossocial" e "alto controle médico", e, que essa abordagem, associou-se também com maior orientação à APS, tendo em vista que os escores significativamente mais altos dos atributos "comunicação interpessoal", "conhecimento do médico sobre o seu paciente" e "coordenação do cuidado" estiveram relacionadas com ela. Da mesma forma, Rohrer et al. ${ }^{13}$, em análise de 680 entrevistas realizadas com pacientes após a consulta com o médico de família e comunidade em Rochester, EUA, mostraram que estar muito satisfeito com fatores da comunicação centrada na pessoa comunicação geral, explicações do médico, escuta, uso de palavras compreensíveis e envolvimento nas decisões - foi positiva e significativamente associado com empoderamento.

Podemos reconhecer limitações no presente estudo. Esta é uma pesquisa avaliativa e transversal, sujeita, assim, às limitações próprias desse tipo de delineamento. Uma delas é a validade externa, já que 
os seus resultados devem ser discutidos mediante a realidade local dos serviços e outra é a impossibilidade de inferência causal em virtude de causalidade reversa, não sendo possível afirmar, assim, se a maior orientação à pessoa faz com que a pessoa fique mais satisfeita com a consulta ou se por estar mais satisfeita a pessoa acabe por avaliar a abordagem do médico como sendo de maior orientação à pessoa. Além disso, o instrumento PPCC também apresenta limitações, sendo a maior, talvez, a que envolve a medida do componente 2 do MCCP, pois, para isso, é usada somente uma questão geral. Por último, o perfil dos médicos envolvidos não foi analisado no presente estudo nem considerado na análise estatística.

\section{Considerações finais}

Aceitando-se a satisfação dos usuários como um desfecho em saúde potencialmente influenciado por muito fatores, entre eles o acesso, a longitudinalidade do cuidado, o tempo de consulta e a relação médico-pessoa ${ }^{6}$-, pode-se agora também compreender o quão fundamental é o papel desempenhado pela abordagem médica na produção de satisfação com a consulta e com os serviços de saúde em pessoas acompanhadas em APS por HAS e/ou DM.

Ou seja, o médico de APS deve ser mais orientado à pessoa, com mais elevada competência cultural, para que possa atingir níveis mais altos de satisfação. Nessa perspectiva, a incorporação da satisfação dos usuários na avaliação dos serviços de saúde deve se constituir em indicador de qualidade, sobretudo, da abordagem praticada pelo médico.

\section{Contribuição dos autores}

Concepção e delineamento do estudo: RCLC, DRK.

Coleta dos dados e construção do banco de dados: RCLC.

Análise e interpretação dos dados: RCLC, DRK.

Redação preliminar: RCLC.

Revisão crítica da versão preliminar: RCLC, DRK.

Todos os autores aprovaram a versão final e concordaram com prestar contas sobre todos os aspectos do trabalho, sendo RCLC E DRK os acrônimos do nome dos autores.

\section{Conflitos de interesse}

Todos os autores declaram não haver conflitos de interesse.

\section{Agradecimento}

Esse estudo obteve recursos financeiros do Programa de Pós-Graduação em Epidemiologia da Faculdade de Medicina da Universidade Federal do Rio Grande do Sul (números de protocolos: 23078.031491/13-49 e 23078.035084/13-47).

\section{Referências}

1. Levenstein JH, McCracken EC, McWhinney IR, Stewart MA, Brown JB. The patient-centred clinical method. 1. a model for the doctorpatient interaction in family medicine. Fam Pract. 1986; 3(1):24-30. 
2. Brown J, Stewart M, McCracken E, McWhinney IR, Levenstein J. The patient-centred clinical method. 2. Definition and application. Fam Pract. 1986 Jun; 3(2):75-9.

3. Stewart M, Brown JB, Weston WW, McWhinney IR, McWilliam CL, Freeman TR. Patient-centered medicine: transforming the clinical method. London: Radcliffe Publishing Ltd; 2014.

4. Esperidião M, Trad L. Avaliação de satisfação de usuários. Ciência \& Saúde Coletiva, 2005; 10(supl.):303-312.

5. Starfield B. Measurement of Outcome: A Proposed Scheme. The Milbank Quarterly. 2005; 83(4):1-11.

6. Sans-Corrales M, Pujol-Ribera E, Gené-Badia J, Pasarín-Rua MI, Iglesias-Peréz B, Casajuana-Brunet J. Family medicine attributes related to satisfaction, health and costs. Family Practice. 2006; 23:308-316.

7. Kloetzel K, Bertoni AM, Irazoqui MC, Campos VP, Santos RN. Controle de qualidade em atenção primária à saúde: a satisfação do usuário. Cad. Saúde Pública. 1998; 14(3):623-628.

8. Trad L, Bastos AC, Santana EM, Nunes M. A construção social da estratégia de saúde da família: condições, sujeitos e contextos. Salvador: PNEPG; 2001.

9. Howard M, Goertzen J, Hutchison B, Kaczorowski J, Morris K. Patient satisfaction with care for urgent health problems: a survey of family practice patients. Ann Fam Med. 2007; (5):419-424.

10. Kinnersley P, Stott N, Peters TJ, Harvey I. The patient-centredness of consultations and outcome in primary care. Br J Gen Pract. 1999 Sep; 49(446):711-6.

11. Little P, Everitt H, Williamson I, Warner G, Moore M, Gould C et al. Observational study of effect of patient centredness and positive approach on outcomes of general practice consultations. BMJ. 2001; 323(7318):908-11.

12. Flocke SA, Miller WL, Crabtree BF. Relationships between physician practice style, patient satisfaction, and attributes of primary care. J Fam Pract. 2002 Oct; 51(10):835-40.

13. Rohrer JE, Wilshusen L, Adamson SC, Merry S. Patient-centredness, self-rated health, and patient empowerment: should providers spend more time communicating with their patients? J Eval Clin Pract. 2008 Aug; 14(4):548-51.

14. Stewart M. What is a successful doctor patient interview? A study of interactions and outcomes. Soc Sci Med. 1984 ; $19: 167-175$.

15. Mead N, Bower P, Hann M. The impact of general practitioners' patient-centredness on patients' post-consultation satisfaction and enablement. Soc Sci Med. 2002 Jul; 55(2):283-99.

16. Bertakis KD, Azari R. Patient-centered care is associated with decreased health care utilization. J Am Board Fam Med. 2011;24(3):229-39.

17. Stewart M, Brown JB, Donner A, McWhinney IR, Oates J, Weston WW et al. The impact of patient centered care on outcomes. J Fam Pract. 2000 Sep; 49(9):796-804.

18. Kolling MG, Harzheim E. Avaliação do método clínico centrado na pessoa: tradução e validação do patient perception of patient centeredness questionnaire [dissertação]. Porto Alegre: Universidade Federal do Rio Grande do Sul; 2012.

19. Beach MC, Price EG, Gary TL, Robinson KA, Gozu A, Palacio A, et al. Cultural competence: a systematic review of health care provider educacional interventions. Med Care. 2005; 43(4):356-73. 